\title{
A TRACE THEOREM FOR CALORIC FUNCTIONS
}

\author{
H.N. MHASKAR \\ Department of Mathematics and Computer Science \\ California State University \\ Los Angeles, California 90032 U.S.A. \\ (Received August 4, 1983)
}

ABSTRACT. Given a solution of the heat equation in an open strip, we state necessary and sufficient conditions for the existence of a boundary function in a given weighted Banach space. We then investigate the relationship between the smoothness of this boundary function and the growth of the solution of the heat equation.

KEY WORDS AND PHRASES. CaZoric functions, moduli of continuity.

1980 MATHEMATCS SUBJECT CLASSIFICATION CODES. 35BG5, 35B30, ¿CAIE

1. INTRODUCTION.

An interesting problem in classical analysis can be described as follows. We are given a sufficiently nice function on an open domain and we wish to extend it to the boundary of the domain while preserving continuity in some sense. When this is possible, we also wish to investigate the relationship between the smoothness of the boundary function and the growth of the function on the domain. For example, we have the following well known theorems in the theory of $\mathrm{H}^{\mathrm{P}}$ spaces:

THEOREM 1. ( $[1]$, p. 33) Let $\mathrm{f}$ be a complex valued harmonic function in the open unit disc $\{z:|z|<1\}$ and set

$$
M_{p}(r, f):= \begin{cases}{\left[\int_{0}^{2 \pi}\left|f\left(r e^{i t}\right)\right|^{p} d t\right]^{1 / p}} & ; 1 \leq p<\infty \\ 0 \leq t \leq 2 \pi & ; p=\infty .\end{cases}
$$

(a) If $1<\mathrm{p} \leq \infty$, then $\mathrm{f}$ is the Poisson integral of a function in $\mathrm{L}^{\mathrm{P}}$ on the unit circle if and on $1 y$ if

$$
\sup _{0<r<1} M_{p}(r, f)<\infty
$$

(b) For $p=1$, $f$ is the Poisson integral of a complex Baire measure on the unit circle if and only if

$$
\sup _{0<r<1} M_{1}(r, f)<\infty .
$$

The following theorem of Hardy and Littlewood relates the smoothness of the boundary function with the growth of the function on the disc. 
THEOREM 2. ( $[2]$, p. 78) Let $1 \leq \mathrm{p}<\infty$, $\mathrm{f}$ be analytic in the open unit disc and(1.2)hold. (Then $f$ is the Poisson integral of a $2 \Pi$-periodic function $g$ in $L^{p}[-\Pi, \Pi]$ ) The following are equivalent:

$$
\begin{aligned}
& \sup _{0<|h| \leq t} \int_{0}^{2 \pi}|g(\theta+h)-g(\theta)|^{p} \text { do }=\sigma\left(t^{\alpha} p\right) \\
& M_{p}\left(r, f^{l}\right)=\alpha(1-r)^{\alpha-i}
\end{aligned}
$$

Here, $0<\propto<1$.

It is well known that caloric functions (i.e. the solutions of the heat equation) share quite a few properties with analytic functions. (e.g [3], [4]). In 1954, Czipszer [5] obtained an analogue of Theorem 1(b) for caloric functions defined on the strip $R \times(0, c)$ where the boundary measure is not necessarily finite itself, but a certain weight function is integrable with respect to the measure. In my dissertation [6], it is demonstrated how the proof of Czipszer can be modified to yield an analogue of Theorem 1(a), not just for $\mathrm{L}^{\mathrm{p}}$ spaces but even for more general weighted rearrangement invariant Banach spaces.

In this paper, we wish to report a somewhat more interesting part of our work in [6], namely, an analogue of Theorem 2. Un1ike the case of analytic functions, the first order modulus of continuity does not seem to be an adequate measurement of the smoothness of the boundary function. Introducing a second order modulus of continuity, we shall obtain a relationship between the growth of the caloric functions and the smoothness of its boundary values.

2. MAIN RESULTS.

Let $X$ be a rearrangement invariant Banach function space. We assume the following: (A) $X$ is isometrically isomorphic to the normed dual of its associate space.

(B) If $f$ is in $X$, then

$$
\lim _{h \rightarrow 0}\|f(x+h)-f(x)\|=0 .
$$

Both the conditions are satisfied for $L^{p}$ spaces if $1<p<\infty$. In general, our theorems will not be true for $p=1, \infty$. With slight modifications, it is possible to obtain the versions valid for the space $C_{0}(R)$. A survey of some of the important properties of the rearrangement invariant Banach function spaces can be found in [7], [ 8 ].

We say that a function $u$ is caloric on $S_{r}:=R \times(0, c)$ if

$$
\frac{\partial^{2} u}{\partial x^{2}}=\frac{\partial u}{\partial t} ;(x, t) \varepsilon S_{c}
$$

For a function $u$ caloric on $S_{c}$, we define the following analogue of the norms $M_{p}$ defined in $(1.1)$ :

$$
N(u, c ; t):=N_{X}(u, c ; t):=(c-t)^{\frac{1}{2}}\|w(c-t, x) u(x, t)\|, 0<t<c
$$
where

$$
w(h, x):=\exp \left(-x^{2} / 4 h\right)
$$

In [6], we proved the following analogue of Theorem $1.1(a)$, the analog'le of Theorem 1.1(b) being given in [5]. 
The following are equivalent:

(a) $u$ is a caloric function on $S_{c}$ and for each $h \varepsilon(0, c)$,

$$
\sup _{0<t<h} N(u, h ; t)<\infty \quad \text {. }
$$

(b) There exists a function $f$ such that $w(h, x) f(x) \varepsilon X$ for each $h \varepsilon(0, c)$ and

$$
u(x, t)=H(f, x, t)
$$

where the operator $\mathrm{H}$ is defined by

$$
H(f, x t, t):=\frac{1}{\sqrt{4 \pi t}} \int w(t, x-y) f(y) d y .
$$

In (2.7) and in all the other formulae of the paper, all integrals are taken over the whole real line unless otherwise specified.

We note that the boundary function $f$ itself is not necessarily in $X$. Because of the presence of the weight functions $w(h, x)$, we need to modify the expression for the usual second order modulus of continuity to measure the smoothness of $f$. In [9], Freud introduced a certain modification which was found to be completely satisfactory for the study of weighted polynomial approximation of such functions. This new modulus is defined as follows. Let $h>0$. With $F(x):=w(h, x) f(x)$, put

$$
\begin{aligned}
\omega_{2}(h, f, \delta):= & \sup _{|t| \leq \delta}|| F(x+2 t)-2 F(x+t)+F(x)|| \\
& +\delta \sup || \zeta_{\delta}(x)[f(x+t)-F(x)]||+\delta^{2}|| \zeta_{\delta}^{2}(x) F(x)||
\end{aligned}
$$

where

$$
\zeta_{\delta}(x):=\min \left\{\delta^{-1},\left(1+x^{2}\right)^{\frac{1}{2}}\right\}
$$

Then the modulus of smoothness of $f$ is given by

$$
\Omega_{2}(h, f, \delta):=\inf _{a, b \in R} \omega_{2}(h, f(x)-a-b x, \delta)
$$

This modulus measures not just the smoothness of $f$ but also the growth of $f$ near $\infty$. In [10] we proved

THEOREM 2. Let $h>0, \delta>0, w(h, x) f(x) \varepsilon X$. Then there exist constants $c_{1}$, $c_{2}$ depending upon $h$ and $X$ alone such that

$$
c_{1} K(h, f, \delta) \leq \Omega_{2}(h, f, \delta) \leq c_{2} K(h, f, \delta)
$$

where the $\mathrm{K}$-functional $\mathrm{K}(\mathrm{h}, \mathrm{f}, \delta)$ is given by

$$
K(h, f, \delta):=\inf \left\{\left\|w(h, x) f_{1}(x)\right\|+\delta^{2}\left\|w(h, x) f_{2}^{\prime \prime}(x)\right\|\right\}
$$

the inf being taken over all $\mathrm{f}_{1}$ and $\mathrm{f}_{2}$ such that $\mathrm{f}=\mathrm{f}_{1}+\mathrm{f}_{2}$, w(h, $\left.\mathrm{x}\right) \mathrm{f}_{1}(\mathrm{x}) \varepsilon \mathrm{X}$, and $\mathrm{f}_{2}$ is a twice iterated integral of a function $\mathrm{f}^{\prime \prime} \varepsilon \mathrm{x}$.

We can now state our main theorem.

THEORFM 3. Let $c>0,0<\propto \leq 1$.

(a) Suppose for each $h \in(0, c), w(h, x) f(x) \varepsilon X$ and

$$
\Omega_{2}(\mathrm{~h}, \mathrm{f}, \delta)=\sigma\left(\delta^{\alpha}\right) \text {. }
$$

Define $u$ by $(2.6)$. Then $u$ is a caloric function on $S_{c}$ satisfying (2.5) and further,

$$
N\left(\frac{\delta u}{\delta t}, h ; t\right)=\sigma\left(t^{\alpha / 2-1}\right) \text { for each } h \varepsilon(0, c) \text { and } \underline{t} \varepsilon(0, h) \text {. }
$$


(b) Let $u$ be a caloric function satisfying (2.5) and (2.14) for each $h \varepsilon(0, c)$ Then there exists a function $f$ such that for every $h \varepsilon(0, c), w(h, x) f(x) \varepsilon X$ and further, $(2.6)$ and $(2.13)$ hold.

3. PROOFS.

We adopt the following notation. $A \ll B$ means that there exists a constant $c$ independent of the obvious variables such that $A \leq c B$. $A \sim B$ means that $A \ll B$ and $B \ll$ A. Thus, in Theorem 2.2,

$$
K(h, f, \delta) \sim \Omega_{2}(h, f, \delta) \text {. }
$$

If $s>0$, I(s) will denote the indicator function of $X$; thus,

$$
I(s):=|f| \sup _{=1}\|\mathrm{f}(\mathrm{st})\| \text {. }
$$

It is known [7] that

$$
\mathrm{I}(\mathrm{s}) \leq \max \left(1, \mathrm{~s}^{-1}\right)
$$

Further, for convenience, we shall often write $w_{h}$ instead of $w(h, \cdot)$. PROOF OF THEOREM 2.3.

(a) We shall prove that $0<\mathrm{t}<\mathrm{h}<\mathrm{h}_{1}<\mathrm{c}$, then

$$
N\left(\frac{\delta u}{\delta t}, h, t\right) \ll t^{-1} \Omega_{2}\left(h_{1}, f ; \sqrt{t}\right) \text {. }
$$

We use Theorem 2.2. Let $\mathrm{f}=\mathrm{g}+\mathrm{f}_{2}$ where $\mathrm{w}_{\mathrm{h}} \mathrm{g} \varepsilon \mathrm{x}, \mathrm{f}_{2}$ is a twice iterated integral of a locally integrable function $\mathrm{f}_{2}^{\prime \prime}$ with $\mathrm{w}_{\mathrm{h}} \mathrm{f}_{2}^{\prime \prime} \varepsilon \mathrm{x}$. Then

$$
u(x, t)=v(x, t)+u_{2}(x, t)
$$

where $v(x, t):=H(g, x, t) ; u_{2}(x, t):=H\left(f_{2}, x, t\right)$. Note that

$$
\frac{\partial u_{2}}{\partial t}=\frac{\partial^{2} u_{2}}{\partial x^{2}}=H\left(f_{2}^{\prime \prime}, x, t\right) .
$$

Hence, in view of the easily verified identity

$$
\begin{aligned}
& \frac{(x-y)^{2}}{t}-\frac{y^{2}}{h}=\frac{h-t}{t h}\left(y-\frac{x h}{h-t}\right)-\frac{x^{2}}{h-t} \\
& \left\|\exp \left(-\frac{x^{2}}{4(h-t)}\right) \frac{\partial u_{2}}{\partial t}\right\| \\
& \leq \sqrt{\frac{h}{h-t}} \| \text { H }\left(\exp \left(-\frac{y^{2}}{4 h}\right) f_{2}^{\prime \prime}(y), x_{h-t} \frac{h}{h-t}\right) \| \\
& \leq \sqrt{\frac{h}{h-t}}|| \exp \left(-\frac{y^{2}}{4 h}\right) f_{2}^{\prime \prime}(y) \| .
\end{aligned}
$$

Thus,

$$
N\left(\frac{\partial u_{2}}{\partial t}, h, t\right)<\left\|\mid w_{h} f_{2}^{\prime \prime}\right\| \leq t^{-1} t\left\|w_{h_{1}} f_{2}^{\prime \prime}\right\| \text {. }
$$

Part (a) will be proved if we now show that

$$
\mathrm{N}\left(\frac{\partial \mathrm{v}}{\partial \mathrm{t}}, \mathrm{h}, \mathrm{t}\right) \ll \mathrm{t}^{-1}\left\|\mathrm{w}_{\mathrm{h}_{1}} \mathrm{f}_{1}\right\| \text {. }
$$


A TRACE THEOREM FOR CALORIC FUNCTIONS

33

Let $0<t<h<h_{1}<c . \quad$ From

$$
v(x, t)=\frac{1}{\sqrt{4 \pi t}} \int \exp \left(-\frac{(x-y)^{2}}{4 t}\right) g(y) d y
$$

we get

$$
\begin{aligned}
& \frac{\partial v}{\partial t}=\frac{\partial^{2} v}{\partial x^{2}}=\frac{1}{\sqrt{4 \pi t}} \int\left[\frac{(x-y)^{2}}{4 t^{2}}-\frac{1}{2 t}\right] \exp \left(\frac{(x-y)^{2}}{4 t}\right) g(y) d y \\
& =\frac{1}{\sqrt{4 \pi t}} \int_{R}\left\{\frac{1}{4 t^{2}}\left[\frac{(h-t)^{2}}{h^{2}}\left(y-\frac{h}{h-t} x\right)^{2}+2 \frac{(h-t) t}{h^{2}} y\left(y-\frac{h}{h-t} x\right)+\frac{t^{2}}{c^{2}} y^{2}\right]\right. \\
& \left.\quad-\frac{1}{2 t}\right\} \exp \left[-\frac{h-t}{4 h t}\left(y-\frac{h}{h-t} x\right)^{2}+\frac{x^{2}}{4(h-t)}\right] \exp -\left(\frac{y^{2}}{4 h}\right) g(y) d y .
\end{aligned}
$$

We shall estimate each term in (3.11) separately.

$\left\|\int_{R}\left(y-\frac{h}{h-t} x\right)^{2} \exp \left[-\frac{h-t}{4 h t}\left(y-\frac{h}{h-t} x\right)^{2}\right] \quad w_{h}(y) g(y) d y\right\|$

$\leq I\left(\frac{h}{h-t}\right) \int_{R} y^{2} \exp \left(-\frac{h-t}{4 h t} y^{2}\right) d y \cdot\left\|w_{h} g\right\|$

$: \quad<\left(\frac{4 h t}{h-t}\right)^{\frac{3}{2}}\left\|w_{h_{1}} g\right\|$

$$
\begin{aligned}
& \left\|\int_{R} y\left(y-\frac{h}{h-t} x\right) \exp \left[-\frac{h-t}{4 h t}\left(y-\frac{h}{h-t} x\right)^{2}\right] w_{h}(y) g(y) d y\right\| \\
& <I\left(\frac{h}{h-t}\right) \int_{R} \exp -\frac{h-t}{4 h t} y^{2} \text { dy }\left\|y^{2} g(y) w_{h}(y)\right\| \\
& <\frac{4 h t}{h-t}\left\|w_{h} g\right\| \\
& \left\|\int_{R} y^{2} \exp \left[-\frac{h-t}{4 h t}\left(y-\frac{h}{h-t} x\right)^{2}\right] g(y) w_{h}(y) d y\right\| \\
& \leq I\left(\frac{h}{h-t}\right) \int_{R} \exp \left(-\frac{h-t}{4 h t} y^{2}\right) d y \cdot\left\|y^{2} g(y) w_{h}(y)\right\| \\
& <\left(\frac{4 h t}{h-t}\right)^{\frac{1}{2}}\left\|w_{h_{1}} g\right\|
\end{aligned}
$$




$$
\begin{aligned}
& \| \int_{R} \exp \left\{-\frac{h-t}{4 h t}\left(y-\frac{h}{h-t} x^{2}\right\} \quad w_{h}(y) g(y) d y \|\right. \\
& \leq I\left(\frac{h}{h-t}\right) \int_{R} \exp \left(-\frac{h-t}{4 h t} y^{2}\right) d y\left\|w_{h} g\right\| \ll\left(\frac{4 h t}{h-t}\right)^{\frac{1}{2}}\left\|w_{h_{1}} g\right\| .
\end{aligned}
$$

Inequality (3.10) follows from $(3.11),(3.12),(3.13),(3.14)$ and (3.15). This completes the proof of part (a).

(b) Choose d small enough so that we can choose $0<h_{1}<\mathrm{h}-2 \mathrm{~d}<\mathrm{c}$.

$$
\begin{aligned}
& \sum_{n=0}^{N}|| \exp \left(-\frac{x^{2}}{4 h_{1}}\right)\left[u\left(x, \frac{d}{2^{n+1}}\right)-u\left(x, \frac{d}{2^{n}}\right)\right] \| \\
& =\sum_{n=0}^{N} \exp \left(-\frac{x^{2}}{4 h_{1}}\right) \int_{d / 2^{n+1}}^{d / 2^{n}} \frac{\partial u}{\partial t}(x, t) d t \| \\
& \leq \sum_{n=0}^{N}\left\|\int_{d / 2^{n+1}}^{d / 2^{n}} \exp -\frac{x^{2}}{4(h-t)}\left|\frac{\partial u}{\partial t}(x, t)\right| d t\right\| \\
& <\sum_{n=0}^{N} \frac{d}{2^{n+1}} \underset{\frac{d}{2^{n+1}} \leq t \leq \frac{d}{2^{n}}}{\sup }\left\|\exp \left(-\frac{x^{2}}{4(h-t)}\right) \frac{\partial u}{\partial t}\right\| \\
& <\sum_{n=0}^{N} \frac{d^{\alpha / 2}}{n \alpha / 2}
\end{aligned}
$$

Thus,

$$
\| \exp \left(-\frac{x^{2}}{4 h_{1}}\right)\left[u\left(x, \frac{d}{2^{N+1}}\right)-u(x, d)\right]=\sigma\left(d^{\alpha / 2}\right)
$$

and the series

$$
\exp \left(-\frac{x^{2}}{4 h}\right)\left\{u,(x, d)+\sum_{n=0}\left[u\left(x, \frac{d}{2^{n+1}}\right)-u\left(x, \frac{d}{2^{n}}\right)\right]\right\}
$$

converges in $X$ to say $\exp \left(-x^{2} / 4 h_{1}\right) f(x)$.

A standard argument then shows that

$$
u(x, t)=\frac{1}{\sqrt{4 \pi t}} \int_{R} w_{t}(x-y) f(y) d y
$$

for all $0<t<h_{1}$ and hence for all $(x, t)$ in $S_{c}$. Since a subsequence of (3.18) can be chosen to converge almost everywhere, $f$ is not dependent upon $h_{1}$ as it might appear to be. Further, from (3.17)

Also,

$$
\left\|w_{h_{1}}(x)[f(x)-u(x, d)]\right\|<d^{\alpha / 2} .
$$

$$
|| w_{h_{1}}(x) \frac{\partial^{2}}{\partial x^{2}} u(x, d) \| \leq|| \exp -\frac{x^{2}}{4(h-d)} \frac{\partial^{2}}{\partial x^{2}} u(x, d)||<d^{\alpha / 2}-1 \text {. }
$$

The proof of part (b) is now complete in view of $(3.20),(3.21)$ and Theorem 2.2. 


\section{REFERENCES}

1. HOFFMAN, K. Banach spaces of analytic functions, Prentice Hall, Englewood Cliffs, N.J., 1962.

2. DUREN, P.L. Theory of $\mathrm{H}^{\mathrm{P}}$ spaces, Academic Press, N.Y., 1970.

3. DOOB, J.L. A probability approach to heat equation, Trans. Amer. Math. Soc., 80 (1955), 216-280.

4. HARTMAN, P. and WINTNER, A. On the solution of the equation of heat conduction, Amer. Jour. of Math., 72 (1950), 359-395.

5. CZIPSZER, J. Hovezetes Vegetelen Rudban, I, Magyar Tudomanyos Akademia Alkalmazolt Matematikai Intezetenek Kolzlemenyei, $\underline{3}$, (1954), 395-405.

6. MHASKAR, H.N. Weighted polynomial approximation on the whole real line and related topics, Ph.D. dissertation, The Ohio State University, Columbus, Ohio, 1980.

7. BENNETT, C. Banach function spaces and interpolation methods, I (The abstract theory), Jour. of Funct. Anal., 17 (1974), 409-440.

8. ZAANEN, A.C. Integration, North Holland Publishers, Amsterdam, 1967.

9. FREUD, G. On the direct and converse theorems in the theory of weighted polynomial approximation, Math. Zeit., 126 (1972), 123-134.

10. FREUD, G. and MHASKAR, H.N. K-functionals and moduli of continuity in weighted polynomial approximation, Arkiv for Mat., 21 (1983), 145-161. 


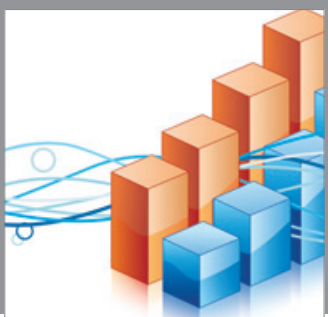

Advances in

Operations Research

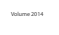

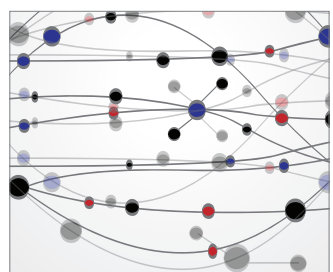

\section{The Scientific} World Journal
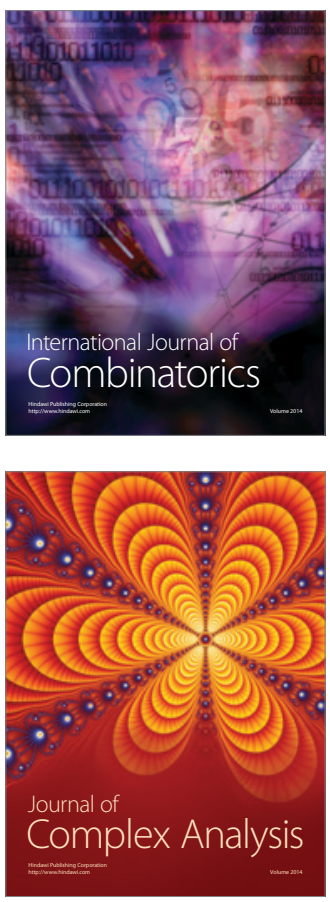

International Journal of

Mathematics and

Mathematical

Sciences
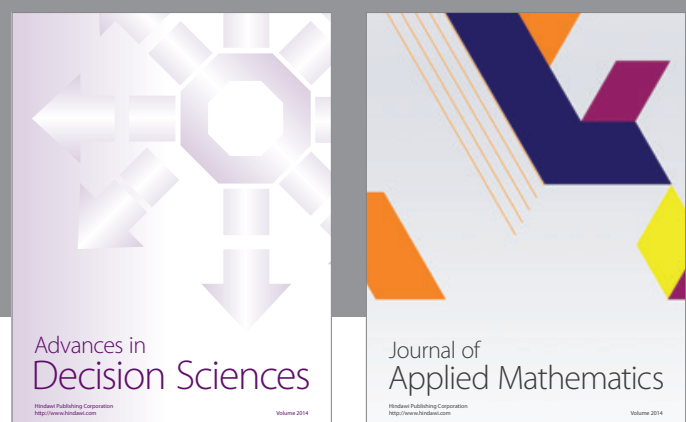

Journal of

Applied Mathematics
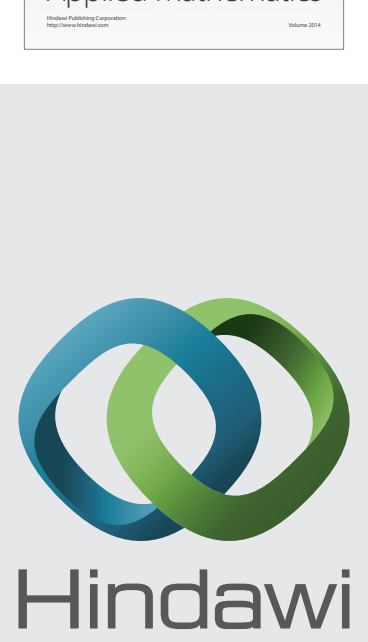

Submit your manuscripts at http://www.hindawi.com
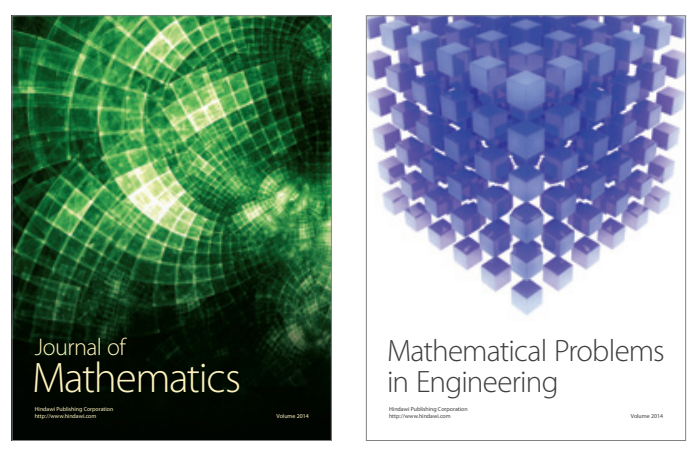

Mathematical Problems in Engineering
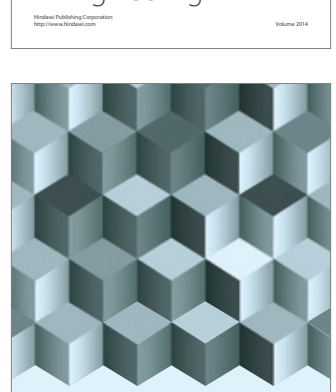

Journal of

Function Spaces
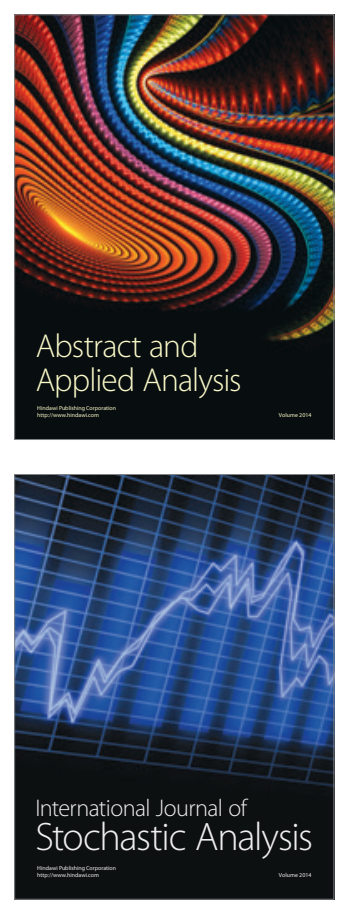

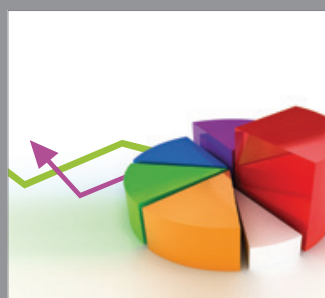

ournal of

Probability and Statistics

Promensencen
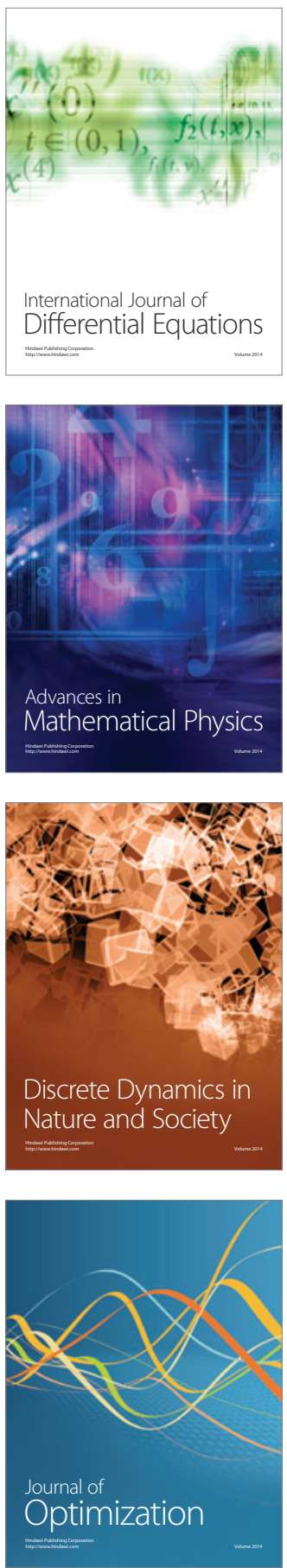